\title{
LABORATORY DIAGNOSIS FOR DENGUE
}

\author{
Marli Tenório CORDEIRO(1)
}

Effective epidemiological surveillance for dengue fever must include the ability of routine laboratory confirmation and monitoring of circulating serotypes. Laboratory surveillance is essential so that differential diagnosis could be performed between dengue fever and other acute febrile diseases, due to clinical similarities exhibited.

In general, the dengue's laboratory diagnosis aims: 1) laboratory, serologic and/or virologic infection's confirmation; 2) identification of circulating serotypes; 3 ) laboratory confirmation of severe and fatal cases; 4) support for epidemiological investigations to determine the levels of disease transmission and 5) performance of molecular studies in order to identify existing genotypes in the region (VORNDAM \& KUNO, 1997). For adequate effectiveness of the laboratory diagnosis, it is required to take into account the appropriate time for blood sampling and to what type of test it is designated. This information is of great importance when interpreting the results.

\section{Laboratory criteria for confirmation of cases}

The positive results for one of the following methods will confirm the case by laboratory criterion: 1 ) isolation and identification of dengue virus (DENV) in blood sample (serum, plasma), organ fragments collected post mortem (liver, spleen, kidney, heart, lung, nervous system); 2) detection of viral nucleic acid (RNA) using the reverse transcription-polymerase chain reaction (RT-PCR) technique; 3) detection of non-structural 1 (NS1) protein (antigen) in serum and/or plasma sample; 4) detection of IgM antibodies specific for dengue in a single serum sample; 5) increase of four times or more in the IgG antibodies titers; hemagglutination inhibiting antibodies, and/or neutralizing antibodies in paired serum samples, collected during the acute phase of illness and convalescence, for one or more DENV antigens; 6) demonstration of viral antigen in necropsied tissues by immunohistochemistry (WHO, 1997; GUZMAN \& KOURI, 2004).

\section{Virologic diagnosis}

For isolation of the virus, the blood collection should be performed preferably within the first five to six days of symptoms, during the acute phase. This sample will also be used for detection of viral RNA by RTPCR, for real time RT-PCR and also for detection of NS1 antigen. Virus isolation is performed usually in cell cultures of mosquito (clone C6/36, Aedes albopictus; TRA-284, Toxorhynchites amboinenses and AP-61,
Aedes pseudoscutellaris), (IGARASHI, 1978). Continuous lines of vertebrates, such as VERO, BHK-21, LLC-MK2, and also inoculation into animals and mosquitos can also be used. The isolated viruses can be identified by indirect immunofluorescence using monoclonal antibodies against all four serotypes (HENCHAL et al., 1982).

\section{Molecular diagnostics by RT-PCR}

The extraction of viral RNA from the samples is carried out using in house techniques (Trisol, Silica/Guanidine isothiocyanate, etc), by column-extraction kits and automated equipment. For detection of viral RNA and identification of serotypes by RT-PCR technique, the protocol described by LANCIOTTI et al. (1992) has been one of the most used in the country. In this technique, universal primers are used for DENV located in the genes C and prM which have good sensitivity and specificity. This fragment is flanked by a sequence conserved among all DENV serotypes allowing genomic amplification. Serotype is then identified by using serotype-specific primers in a semi-nested PCR in a second amplification. The RT-PCR products (cDNA) can be viewed by $1 \%$ agarose gel electrophoresis, and then digitalized.

Real time RT-PCR, in addition to be more sensitive, has the advantage to be able to process a large number of samples at once, and be used both qualitatively and quantitatively (determination of viral load).

The use of conventional RT-PCR in early diagnosis of suspected cases and to monitor viral circulation has proven to be a valuable diagnostic tool, with the advantage of not providing significant difference in sensitivity, in both primary and secondary cases, or even in the presence or absence of IgM in the serum sample tested (CORDEIRO et al., 2007).

\section{Detection of NS1 antigen}

The hexameric form of NS1 protein is highly conserved in all four DENV serotypes and was found circulating in the blood of patients from the first to the ninth day after the onset of fever (YOUNG et al., 2000). Because it is present in the serum during the acute phase of the infection, NS1 antigen is a marker used in the early diagnosis of the disease, with a greater sensitivity in the first five days of disease. Detection of NS1 by ELISA is rapid and as sensitive as RT-PCR, but does not distinguish the virus serotypes. 
Moreover, the sensitivity of the test can vary depending on the type of infection. Results obtained in our studies using kit Platelia ${ }^{\mathrm{TM}}$ Dengue NS1Ag-ELISA (BIORAD) demonstrated greater sensitivity in the confirmation of primary cases $(94.0 \%$; 94/100), compared to secondary ones $(54.1 \% ; 66 / 122)(\mathrm{p}<0.0001)$. Sensitivities of $97.5 \%$ and $59.1 \%$ for primary and secondary infections, respectively, were also observed by McBRIDE (2009).

False-negative results can occur, and possibly result from the formation of immune complexes of NS1 antigen with IgG, particularly in secondary infections, where target antigens are no longer accessible to the monoclonal antibody from ELISA (HANG et al., 2009). Because NS1 capture test is more effective in the acute phase of the disease, this could be used in combination with ELISA-IgM to increase detection sensitivity, particularly in areas of high prevalence of secondary infections.

\section{Serological diagnosis}

Serological diagnosis detects specific antibodies to the virus and complements the virological diagnosis, and when this is not possible, it works as an alternative way for diagnosis. The detection of antibodies can be made using immunoenzymatic (ELISA) and immunochromatographic techniques; test for hemagglutination inhibition (HI), plaque reduction neutralization test (PRNT), etc.

Specific antibodies IgM are produced temporarily, during both primary and secondary infection. Anti-dengue $\operatorname{IgM}$ can be detected, usually from the fifth day of disease and may persist for 60 to 90 days. Therefore, its detection in any serum sample is indicative of active or recent infection, within the last two to three months. The production of antibodies for DENV is usually distinct from those having primary and secondary infections. In the primary infection, $\operatorname{IgM}$ is usually detected from the fifth day of disease, while IgG is detected at low levels, generally from the seventh day after the onset of infection. Conversely, in the secondary infection, antibodies IgG are detected at high levels in acute phase, while antibodies IgM are usually detected in the lower titers than those observed in the primary infections (GUZMAN \& KOURI, 2004).

Importantly, during a secondary infection (sequential), about $20 \%$ of patients do not have IgM at detectable levels and, therefore, it is required to perform other serological tests, such as hemagglutination inhibition test or plaque reduction neutralization test, to confirm the case (VORNDAM \& KUNO, 1997).

In the primary infection, relatively monotypic neutralizing antibodies are detected. In the secondary infections, high titers of neutralizing antibodies are produced to two or more of the four DENV serotypes. In some combinations of sequential infections, the highest titer for neutralizing antibodies in the serum of convalescent patient is targeted against the virus that infected earlier, a phenomenon called "original antigenic sin", which can sometimes hamper the interpretation of the serological test (VORNDAM \& KUNO, 1997).

For detection of antibodies class IgM, IgM-capture immunoenzymatic technique (MAC-ELISA), developed by KUNO et al. (1987) can be used, as well as the immunoenzymatic kits (ELISA) for IgM anti-dengue capture, provided that they have good sensitivity and specificity. For detection of antibodies IgG anti-dengue, both in-house techniques (GAC-
ELISA) and commercial kits for IgG-capture or indirect ones validated by reference centers can be used.

For the quantification of hemagglutination inhibition antibodies (total: $\operatorname{IgM}$ and $\operatorname{IgG}$ ), Hemagglutination Inhibition technique in microtechnique, adapted by CLARKE \& CASALS (1958) can be used and this also serves to characterize the type of immune response, according to WHO criteria (CORDEIRO et al., 2007).

The neutralizing test by plaque reduction is the method considered "gold-standard" to determine DENV serotype-specific immunity, although new techniques to measure neutralizing antibodies are currently under development. This technique is recommended by the World Health Organization to assess the effectiveness of dengue vaccines in development (ROEHRING et al., 2008).

The neutralizing activity in serum is determined by their ability to reduce the largest number of viral plaques. The positive sample is determined by a reduction of $50 \%$ or more in plaque formation (MORENS, 1985). Variations in the implementation of PRNT and the lack of standardization of the technique, which allows the use of a wide variety of cell lines, viral isolated from different sources, different cell culture media, etc., represent a limitation of the technique, and may produce different results, hampering the comparison of results between laboratories (ROEHRING et al., 2008). To circumvent this problem, WHO issued a guidance with recommendations to perform PRNT (WHO, 2007).

\section{CONCLUSION}

The results obtained with different techniques available for laboratory diagnosis of suspected dengue cases analyzed in our laboratory allowed us to observe significant differences in the sensitivity of some tests, some related to the time of illness of the patient, others with the characteristics of the infection (primary or secondary), presence or absence of $\operatorname{IgM}$ in the sample, etc. Thus, when a case of dengue is desired to be confirmed through laboratory tests, it is often required to use several techniques currently available and not restrict to a single methodology.

\section{REFERENCES}

1. Clarke DH, Casals J. Techniques for hemagglutination and hemagglutination-inhibition with arthropod-borne viruses. Am J Trop Med Hyg. 1958;7:561-73.

2. Cordeiro MT, Silva AM, Brito CA, Nascimento EJ, Magalhães MC, Guimarães GF, et al. Characterization of a dengue patient cohort in Recife, Brazil. Am J Trop Med Hyg. 2007;77:3328-34.

3. Guzman MG, Kouri G. Dengue diagnosis, advances and challenges. Int J Infect Dis. 2004;8:69-80.

4. Hang VT, Nguyet NM, Trung DT, Tricou V, Yoksan S, Dung NM, et al. Diagnostic accuracy of NS1 ELISA and lateral flow rapid tests for dengue sensitivity, specificity and relationship to viraemia and antibody responses. PloS Negl Trop Dis. 2009;3:e360.

5. Henchal EA, Gentry MK, McCown JM, Brandt W E. Dengue virus-specific and flavivirus group determinants identified with monoclonal antibodies by indirect immunofluorescence. Am J Trop Med Hyg. 1982;31:830-6.

6. Igarashi A. Isolation of a Singh's Aedes albopicitus cell clone sensitive to dengue and chikungunya viruses. J GenVirol. 1978;40:531-44. 
7. Kuno G, Gomez I, Gubler DJ. Detecting artificial anti-dengue IgM immune complexes using an enzyme-linked immunosorbent assay. Am J Trop Med Hyg. 1987;36:153-9.

8. Lanciotti RS, Calisher CH, Gubler DJ, Chang GJ, Vorndam AV. Rapid detection and typing of dengue viruses from clinical samples by using Reverse Transcriptase Polymerase Chain Reaction. J Clin Microbiol. 1992;30:545-51.

9. McBride WJ. Evaluation of dengue NS1 test kits for the diagnosis of dengue fever. Diagn Microbiol Infect Dis. 2009;64:39-44.

10. Morens DM. Simplified plaque reduction neutralization assay for dengue viruses by semimicro methods in BHK-21 cells: comparison of BHK suspension test with standard plaque reduction neutralization. J Clin Microbiol. 1985;22:250-4.

11. Roehring JT, Hombach J, Barrett ADT. Guidelines for plaque-reduction neutralization testing of human antibodies to dengue viruses. Viral Immunol. 2008;21:123-32.
12. Vorndam V, Kuno G. Laboratory diagnosis of dengue virus infection. In: Gubler DJ, Kuno G, editors. Dengue and dengue hemorrhagic fever. New York: CAB International; 1997. p. 313-33.

13. World Health Organization. Dengue hemorrhagic fever: diagnosis, treatment, prevention and control. $2^{\text {nd }}$ ed. Geneva: WHO; 1997.

14. World Health Organization. Guidelines for plaque reduction neutralization testing of human antibodies to dengue viruses. 2007. Available from: <http://www.who.int/ mediacentre/>.

15. Young PR, Hilditch PA, Bletchly C, Halloran W. An antigen capture enzyme-linked immunosorbent assay reveals high levels of the dengue virus protein NS1 in the sera of infected patients. J Clin Microbiol. 2000;38:1053-7. 